\title{
Risk Factors for Acute Cholangitis Caused by Enterococcus faecalis and Enterococcus faecium
}

\author{
Yuki Karasawa ${ }^{1}$, Jun Kato ${ }^{1,2,3,4}$, Satoshi Kawamura ${ }^{5}$, Kentaro Kojima ${ }^{1}$, Takamasa Ohki ${ }^{1}$, Michiharu Seki ${ }^{1}$, Kazumi \\ Tagawa ${ }^{1}$, and Nobuo Toda ${ }^{1,2}$ \\ ${ }^{1}$ Department of Gastroenterology and ${ }^{2}$ Center of Endoscopy, Mitsui Memorial Hospital, Tokyo, ${ }^{3}$ Department of Gastroenterology and \\ ${ }^{4}$ Center of Endoscopy, Chiba University Hospital, Chiba, and ${ }^{5}$ Department of Gastroenterology, University of Tokyo Hospital, Tokyo, \\ Japan
}

\section{Article Info}

Received July 11, 2020

Revised September 25, 2020

Accepted October 5, 2020

Published online December 31, 2020

\section{Corresponding Author}

Nobuo Toda

ORCID https://orcid.org/0000-0001-5426-1896

E-mail ntoda@mitsuihosp.or.jp

\begin{abstract}
Background/Aims: Acute cholangitis (AC) is a potentially life-threatening bacterial infection, and timely antimicrobial treatment, faster than that achieved with bacterial cultures, is recommended. Although the current guidelines refer to empirical antimicrobial treatment, various kinds of antimicrobial agents have been cited because of insufficient analyses on the spectrum of pathogens in AC. Enterococcus spp. is one of the most frequently isolated Gram-positive bacteria from the bile of patients with $\mathrm{AC}$, but its risk factors have not been extensively studied. This study aimed to analyze the risk factors of $\mathrm{AC}$ caused by Enterococcus faecalis and Enterococcus faecium.
\end{abstract}

Methods: Patients with AC who were hospitalized in a Japanese tertiary center between 2010 and 2015 were retrospectively analyzed. Patients' first AC episodes in the hospital were evaluated.

Results: A total of 266 patients with $A C$ were identified. E. faecalis and/or E. faecium was isolated in $56(21 \%)$ episodes of AC. Prior endoscopic sphincterotomy (EST), the presence of a biliary stent, prior cholecystectomy, and past intensive care unit admission were more frequently observed in AC patients with $E$. faecalis and/or $E$. faecium than in those without such bacteria. Prior EST was identified as an independent risk factor for AC caused by $E$. faecalis and/or $E$. faecium in the multivariate analysis.

Conclusions: Given the intrinsic resistance of $E$. faecalis and $E$. faecium to antibiotics, clinicians should consider empirical therapy with anti-enterococcal antibiotics for patients with prior EST. (Gut Liver 2021;15:616-624)

Key Words: Cholangitis; Enterococcus; Sphincterotomy; Anti-microbial agents; Therapeutics

\section{INTRODUCTION}

Acute cholangitis (AC) is a potentially life-threatening bacterial infection of the intra- and/or extrahepatic biliary system caused by obstruction of the bile ducts, with stasis and subsequent infection of the bile. ${ }^{1}$ The common causes of AC include gallstones, bile duct stones, and bile duct stenosis in cases of chronic pancreatitis, malignant neoplasm, and sclerosing cholangitis. ${ }^{2,3}$ The typical symptoms of AC are fever, jaundice, and abdominal pain (Charcot's triad). ${ }^{4}$ The current treatment strategies support a risk-stratified approach based on the revised Tokyo Guidelines 2018
(TG18) and generally comprise a combination of antibiotic therapy and early endoscopic resolution of the obstruction, ${ }^{5,6}$ because delaying the endoscopic treatment often results in persistent organ failure. ${ }^{7}$

Pathogens most frequently isolated from the bile of patients with AC are Gram-negative Enterobacteriaceae, such as Escherichia coli and Klebsiella spp., as well as grampositive Enterococcus spp., with a high proportion of polymicrobial cultures observed. ${ }^{8,9}$ Given that microbiological identification of the pathogens requires time, antibiotic therapy is generally initiated as an empirical therapy. The current guidelines, such as TG18, classify antimicrobial 
recommendations according to the route of infection (community-acquired or healthcare-associated) and grade of AC. Many types of antibiotics, including any generation of cephalosporins, are recommended in the guidelines, and no priority is indicated. ${ }^{10}$

Enterococci, which accounts for a considerable portion of pathogens causing $\mathrm{AC}$, are potentially resistant to cephalosporins. According to the Clinical and Laboratory Standards Institute guidelines, there are no breakpoints of ceftriaxone for Enterococcus spp. available. Susceptibility to fourth-generation cephalosporins (cefpirome and cefozopran) was reported to be $60 \%$ for Enterococcus faecalis and $15 \%$ for Enterococcus faecium.

In addition, vancomycin-resistant enterococci (VRE) are becoming an increasingly important cause of invasive infections in the United States. ${ }^{11,12}$ The most common type of vancomycin resistance in enterococci is associated with acquisition of the van A and van B genes, typically observed in E. faecalis and $E$. faecium isolates. ${ }^{13}$ These species comprise the majority of VRE isolated from blood stream infections and are associated with significant mortality. ${ }^{14}$

In earlier studies, Enterococcus spp. accounted for $40.5 \%$ of all identified pathogens. ${ }^{15}$ Moreover, Enterococcus spp. constituted $43 \%$ of all pathogens isolated from patients with AC. ${ }^{16}$ However, knowledge of the risk factors of AC with Enterococcus spp. is limited. The aims of this retrospective study were to characterize the contemporary microbial patterns in patients with AC using a pathogenbased approach and to identify the risk factors for AC caused by $E$. faecalis and/or E. faecium.

\section{MATERIALS AND METHODS}

\section{Study design}

The medical records of patients who were admitted to Mitsui Memorial Hospital, a Japanese tertiary center, from January 2010 to December 2015 were reviewed to identify patients with AC. All patients, regardless of age, whose blood and/or bile cultures had been obtained were included in our analysis. We defined AC based on TG18. Even if neither blood nor bile cultures were positive, the episodes that met the criteria of TG18 and were clinically consistent with AC were enrolled in this study. We excluded patients with other infectious diseases.

The following variables were collected from the medical records: age; sex; microbiological results of blood and/or bile cultures; underlying biliary disease (common bile duct calculus, gallstone or biliary sludge, etc.); medications, including any antibiotic used within 14 days prior to the occurrence of cholangitis; admission to the intensive care unit; previous endoscopic diagnostic procedures or interventions; and history of biliary or upper gastrointestinal surgery. We also classified the severity of each case according to TG18. ${ }^{5}$ As the outcomes of cholangitis, length of hospital stay and mortality were also examined. In patients who had two or more episodes of AC during the study period, the first episode alone was included in the analysis. The development of cholangitis during the hospital stay for $\geq 48$ hours was regarded as nosocomial AC.

This study was approved by the Ethical Committee of Mitsui Memorial Hospital (IRB number: MEC2018-C12). The requirement for obtaining informed consent from the patients was waived due to the retrospective nature of the study.

\section{Microbiological sampling}

Blood culture samples were collected before or immediately after the initiation of antibiotic treatment, followed by bedside inoculation of $10 \mathrm{~mL}$ of blood into the blood culture bottles (BacT/Alert; bioMerieux, Durham, NC, USA). The bottles were incubated at $37^{\circ} \mathrm{C}$ until microbial growth was detected, or for at least 7 days. Bile cultures were obtained by endoscopic retrograde cholangiopancreatography via catheter aspiration. Bile cultures were performed using standard solid media, e.g., sheep blood agar (Kyokuto Pharmaceutical Industrial Co., Ltd., Tokyo, Japan) for aerobic bacteria and Brucella agar (Kyokuto Pharmaceutical Industrial Co., Ltd.) for anaerobic bacteria. The cultivated microorganisms were identified, and antibiotic susceptibility testing was performed using the MicroScan WalkAway (Beckman Coulter, Brea, CA, USA), with the results interpreted according to the Clinical and Laboratory Standards Institute guidelines.

\section{Statistical analysis}

Statistical analysis was performed using JMP 11.0.0 (SAS Institute Inc., Cary, NC, USA). Significant differences were assessed using the Pearson chi-square test. The risk factors for cholangitis caused by $E$. faecalis and/or E. faecium were determined by performing a multivariate binary logistic regression analysis, including the use of significant univariate predictors through stepwise backward elimination. The significance level in two-sided testing was $\mathrm{p}<0.05$, without correction for multiple testing. We also compared the sensitivity of bile and blood cultures with Wilcoxon signed-rank test.

\section{RESULTS}

\section{Baseline characteristics}

During the study period, 266 patients who developed 
one or more episodes of AC were identified. The analysis in this study was based on the first AC episode of each patient. Of the 266 patients, 174 (65\%) were males. The median length of hospital stay was 16 days, and the 30day mortality rate was $2.8 \%$. Ten patients $(4 \%)$ were in septic shock, and eight patients (3\%) required inotropes. In 42 patients ( $16 \%$ ), the onset was $>48$ hours after hospital admission and was regarded as nosocomial AC. Common bile duct calculus was observed in 143 patients (54\%), whereas 150 patients (56\%) had gallstone or biliary sludge. We investigated the patients' comorbidities that are known to suppress immunity, such as renal failure treated with hemodialysis (nine patients, $3 \%$ ), diabetes mellitus (60 patients, $23 \%$ ), and liver cirrhosis (10 patients, $4 \%$ ). Prior biliary interventions, including endoscopic sphincterotomy (EST) (60 patients, 23\%) and endoscopic papillary balloon dilation (EPBD) (15 patients, 6\%), were evaluated (Table 1).

\section{Overall pathogen spectra}

From the 266 AC cases, 230 bile cultures and 239 blood cultures were obtained, and positive results were noted in 131 (57\%) and 155 (65\%) episodes, respectively. Blood and/or bile cultures were positive in 183 episodes (69\%) and were negative in the remaining 83 episodes (31\%). E. faecalis and/or $E$. faecium were identified in 56 cases (21\%). Enterococcus spp. other than $E$. faecium or $E$. faecalis were observed in 19 cases (7\%), and bacteria other than enterococci alone were isolated in 108 cases (41\%) (Fig. 1).

The overall bacterial spectrum revealed that, among 75 cases with Enterococcus spp., the most frequently identified species was $E$. faecalis $(33 / 266,12.4 \%)$, followed by $E$. faecium (26/266, 9.8\%) and E. casseliflavus (18/266, 6.8\%) (Table 2, Fig. 2). Among species other than the Enterococcus spp., E. coli was the most frequently isolated pathogen $(72 / 266,27.2 \%)$, followed by Klebsiella pneumoniae (47/266, 17.7\%). The isolated bacterial spectrum did not differ significantly according to severity of AC (Table 3 ). In addition, there was no significant difference in sensitivity between bile and blood cultures ( $\mathrm{p}=0.235$ ).

\section{Comparison of characteristics between $A C$ patients with and without $E$. faecalis and/or $E$. faecium}

To identify the risk factors of AC caused by $E$. faecalis and/or $E$. faecium, univariate comparisons were performed between patients with and without these bacteria (Table 4). There was a significant difference in the frequency of prior EST between patients with $\mathrm{AC}$ with and without $E$. faecalis and/or E. faecium (24/56 [43\%] vs 36/210 [17\%]; p<0.001). In addition, a statistical difference was observed in the frequency of the presence of biliary stent (16/56 [29\%] vs 28/210 [13\%]; p=0.006), prior cholecystectomy (14/56 [25\%]
Table 1. Characteristics of the Studied Patients

\begin{tabular}{|c|c|}
\hline Characteristics & №. $(\%)$ \\
\hline \multicolumn{2}{|l|}{ Patients } \\
\hline Number & 266 \\
\hline Age at onset, median (range), yr & 74 (29-97) \\
\hline Age $\geq 75 y r$ & $131(49)$ \\
\hline \multicolumn{2}{|l|}{ Sex } \\
\hline Male & $174(65)$ \\
\hline Female & 92 (34) \\
\hline Length of hospital stay, median (range), day & $16(2-184)$ \\
\hline 30-Day mortality rates, $\%$ & 2.8 \\
\hline Septic shock & $10(4)$ \\
\hline Usage of inotropes & $8(3)$ \\
\hline Onset of $>48$ hours after admission & $42(16)$ \\
\hline \multicolumn{2}{|l|}{ Underlying biliary disease } \\
\hline Common bile duct calculus & $143(54)$ \\
\hline Gallstone or biliary sludge & $150(56)$ \\
\hline Malignant neoplasm of biliary tract & $95(36)$ \\
\hline Cholecystitis & $22(8)$ \\
\hline Hemobilia & $3(1)$ \\
\hline \multicolumn{2}{|l|}{ Comorbidity } \\
\hline Renal failure treated with hemodialysis & 9 (3) \\
\hline Diabetes mellitus & $60(23)$ \\
\hline Liver cirrhosis & $10(4)$ \\
\hline \multicolumn{2}{|l|}{ Prior biliary intervention } \\
\hline Endoscopic sphincterotomy & $60(23)$ \\
\hline Endoscopic papillary balloon dilation & $15(6)$ \\
\hline Biliary stent & $44(17)$ \\
\hline Percutaneous transhepatic cholangiodrainage & $14(5)$ \\
\hline PTGBA/PTGBD & 7 (3) \\
\hline Biliary tract reconstruction & $25(9)$ \\
\hline Cholecystectomy & $42(16)$ \\
\hline \multicolumn{2}{|l|}{ Prior anastomosis in upper $\mathrm{Gl}$} \\
\hline Billroth II & $5(2)$ \\
\hline Roux-en-Y anastomosis & $10(4)$ \\
\hline \multicolumn{2}{|l|}{ ICU admission } \\
\hline None & $109(41)$ \\
\hline Past & $61(23)$ \\
\hline Current & $123(46)$ \\
\hline $\begin{array}{l}\text { Antibiotic use within } 14 \text { days prior to occurrence of } \\
\text { cholangitis }\end{array}$ & $47(18)$ \\
\hline Cephem & $30(11)$ \\
\hline 1st generation & $1(0.4)$ \\
\hline 2nd generation & $19(29)$ \\
\hline 3rd generation & $10(4)$ \\
\hline 4th generation & 0 \\
\hline Penicillin & $3(1)$ \\
\hline Carbapenem & $5(2)$ \\
\hline
\end{tabular}

PTGBA/PTGBD, percutaneous transhepatic gallbladder aspiration/ drainage; GI, gastrointestinal tract; ICU, intensive care unit.

vs $28 / 210$ [13\%]; $\mathrm{p}=0.03$ ), and past intensive care unit admission (19/56 [34\%] vs $42 / 210$ [20\%]; $\mathrm{p}=0.03)$ between the two groups (Table 4). No statistical difference was observed in age, sex, septic shock, inotrope use, malignant neoplasm of the biliary tract, cholecystitis, comorbidity (e.g., renal failure, diabetes mellitus, and liver cirrhosis), biliary tract reconstruction, prior anastomosis in the upper gastrointestinal 


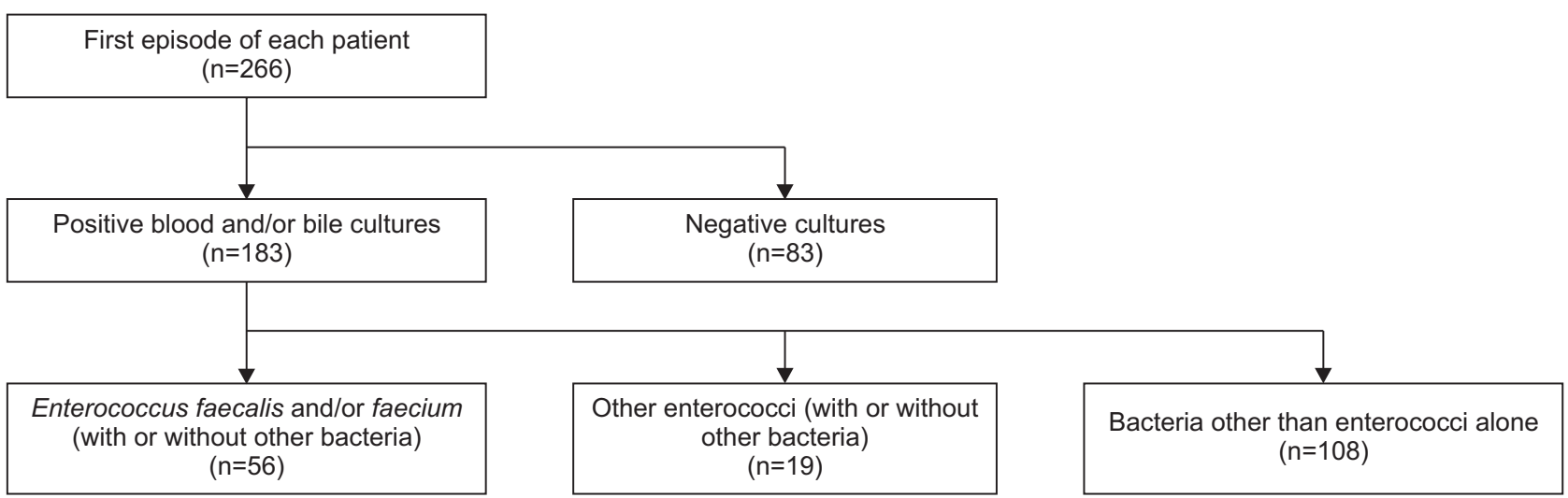

Fig. 1. Study flowchart.

Table 2. Bacteria Isolated from Bile and/or Blood Cultures Obtained from Patients with Cholangitis

\begin{tabular}{lccr}
\hline \multicolumn{1}{c}{ Bacterial species } & Total $(n=266)$ & $\begin{array}{c}\text { Bile culture }(n=230), \\
\text { positive }(n=131,57 \%)\end{array}$ & $\begin{array}{c}\text { Blood culture }(n=239), \\
\text { positive }(n=155,65 \%)\end{array}$ \\
\hline Enterococcus spp. & & & $16(7)$ \\
Enterococcus faecalis & $33(12)$ & $27(12)$ & $18(8)$ \\
Enterococcus faecium & $26(10)$ & $17(7)$ & $11(5)$ \\
Enterococcus casseliflavus & $18(7)$ & $11(5)$ & $3(1)$ \\
Enterococcus avium & $5(2)$ & $5(2)$ & $1(0)$ \\
Enterococcus raffinosus & $2(1)$ & $2(1)$ & $2(1)$ \\
Enterococcus gallinarum & $4(2)$ & $2(1)$ & $49(21)$ \\
Others & & & $26(11)$ \\
Escherichia coli & $72(27)$ & $44(19)$ & $12(5)$ \\
Klebsiella pneumoniae & $47(18)$ & $36(17)$ & $6(3)$ \\
Klebsiella oxytoca & $19(7)$ & $13(6)$ & $10(4)$ \\
Enterobacter cloacae & $12(5)$ & $9(4)$ & $6(3)$ \\
Clostridium perfringens & $17(6)$ & $15(7)$ & $4(2)$ \\
Aeromonas hydrophila & $17(6)$ & $13(6)$ & $11(5)$ \\
Bacteroides fragilis & $8(3)$ & $7(3)$ & $6(3)$ \\
Pseudomonas aeruginosa & $24(9)$ & $22(10)$ & \\
Staphylococcus epidermidis & $9(3)$ & $4(2)$ & \\
\hline
\end{tabular}

Data are presented as the number (\%). Cases which were caused by multiple microorganisms were counted separately.

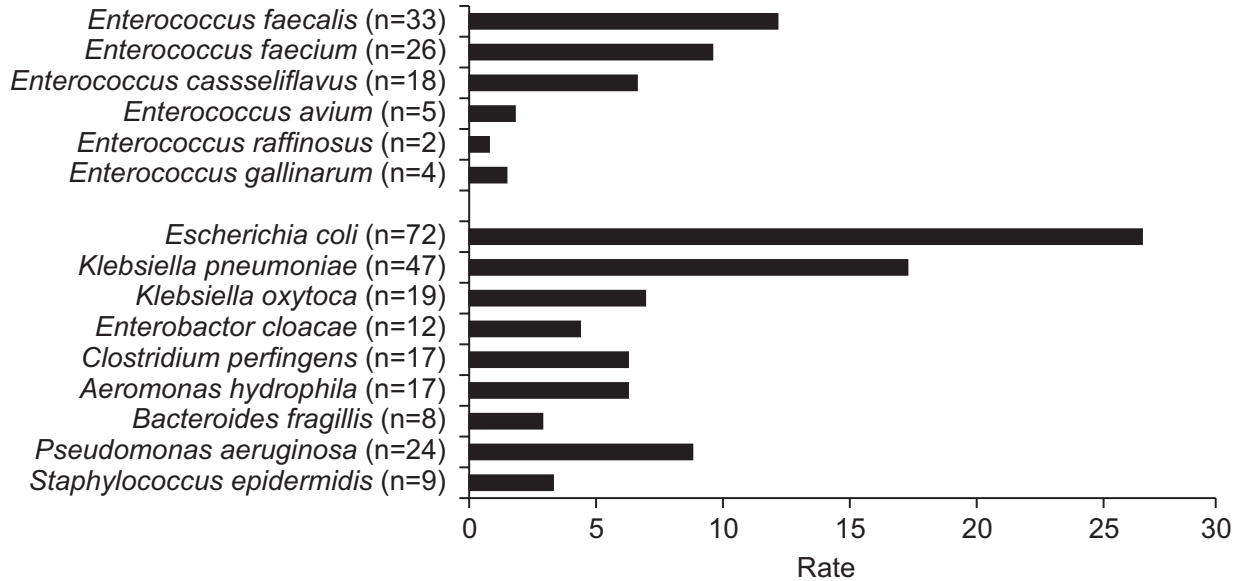

Enterococcus faecalis $(\mathrm{n}=33)$ Enterococcus faecium $(n=26)$ Enterococcus avium $(\mathrm{n}=5)$ Enterococcus raffinosus $(\mathrm{n}=2)$ Escherichia coli $(\mathrm{n}=72)$ ( monas hydrophila $(n=17)$ Bacteroides fragillis $(\mathrm{n}=8)$ Pseudomonas aeruginosa $(\mathrm{n}=24)$ Staphylococcus epidermidis $(n=9)$

Rate
Fig. 2. Pathogen spectra in the blood and/or bile cultures of the included patients. tract, or antibiotic use within 14 days prior to the occurrence of cholangitis in the univariate logistic regression analysis (all $\mathrm{p}>0.05)$.
The multivariate analysis, including prior EST, biliary stent, cholecystectomy, and past ICU admission as variables, revealed that prior EST was the only independent 
Table 3. Comparison of Pathogens Obtained from Blood and/or Bile Cultures and 30-Day Mortality Rates among Each Severity Grade

\begin{tabular}{|c|c|c|c|c|}
\hline \multirow{2}{*}{ Bacterial species } & \multirow{2}{*}{ Total $(n=266)$} & \multicolumn{3}{|c|}{ Severity* } \\
\hline & & Grade I ( $n=129,48 \%)$ & Grade II (n=58, 22\%) & Grade III (n=79, 30\%) \\
\hline \multicolumn{5}{|l|}{ Enterococcus spp. } \\
\hline Enterococcus faecalis & $33(12)$ & $15(12)$ & $8(14)$ & 10 (13) \\
\hline Enterococcus faecium & $26(10)$ & $11(9)$ & $5(9)$ & $10(13)$ \\
\hline Enterococcus casseliflavus & $18(7)$ & $10(8)$ & 2 (3) & $6(8)$ \\
\hline Enterococcus avium & $5(2)$ & $1(1)$ & 2 (3) & 2 (3) \\
\hline Enterococcus raffinosus & $2(1)$ & $2(2)$ & 0 & 0 \\
\hline Enterococcus gallinarum & $4(2)$ & $2(2)$ & 2 (3) & 0 \\
\hline \multicolumn{5}{|l|}{ Others } \\
\hline Escherichia coli & $72(27)$ & $28(22)$ & $16(28)$ & $28(35)$ \\
\hline Klebsiella pneumoniae & $47(18)$ & $18(14)$ & $13(22)$ & $16(20)$ \\
\hline Klebsiella oxytoca & $19(7)$ & $8(6)$ & $5(9)$ & $5(6)$ \\
\hline Enterobacter cloacae & $12(5)$ & $7(5)$ & 2 (3) & $3(4)$ \\
\hline Clostridium perfringens & $17(6)$ & $8(6)$ & 2 (3) & $7(9)$ \\
\hline Aeromonas hydrophila & $17(6)$ & $5(4)$ & $6(10)$ & $6(8)$ \\
\hline Bacteroides fragilis & $8(3)$ & $1(1)$ & $3(5)$ & $4(5)$ \\
\hline Pseudomonas aeruginosa & $24(9)$ & $14(11)$ & $3(5)$ & 7 (9) \\
\hline Staphylococcus epidermidis & 9 (3) & $8(6)$ & 0 & $1(1)$ \\
\hline 30-Day mortality rates, \% & 2.8 & 2.5 & 1.9 & 3.9 \\
\hline
\end{tabular}

Data are presented as the number (\%). Cases that were caused by multiple microorganisms were counted separately.

*Severity of acute cholangitis was defined according to the Tokyo Guidelines 2018.

predictor of AC caused by $E$. faecalis and/or E. faecium (odds ratio, 3.53; 95\% confidence interval, 1.84 to 6.78 ; $\mathrm{p}<0.001$ ) (Table 4).

\section{DISCUSSION}

The current guidelines, such as TG18, recommend the combination of antimicrobial agents, including vancomycin, to cover treatment of Enterococcus spp. in grade III community-acquired AC and healthcare-associated acute biliary infections. In contrast, they indicated that cephalosporin-based therapy (each generation of cephalosporin \pm metronidazole) can also be chosen for grade I or II community-acquired AC. Although TG18 determined whether Enterococcus spp. should be covered or not according to the grade of AC, the current study revealed that prior EST is the only independent risk factor of AC caused by $E$. faecalis and/or E. faecium, regardless of the grade.

Enterococcus spp. show intrinsic resistance to any generation of cephalosporins. E. faecalis is usually the most frequently isolated Enterococcus spp. from human clinical specimens, representing $80 \%$ to $90 \%$ of the isolates in Enterococcus spp., followed by $E$. faecium, which accounts for $5 \%$ to $10 \%$ of all enterococcal infections. ${ }^{17-20}$ A previous study reported that, of the 826 unique episodes of bacteremia with Enterococcus spp., $94.6 \%$ were caused by either $E$. faecalis (56.1\%) or E. faecium (38.5\%). ${ }^{21}$

Moreover, VRE currently account for $>30 \%$ of entero- coccal infections, ${ }^{22}$ and more than $90 \%$ of VRE isolates in the United States are E. faecium..$^{22}$ In the present study, VRE were not isolated since VRE infections are less frequent in Japan than in the United States. However, E. faecalis and E. faecium are potentially important in terms of antibiotic resistance. The susceptibility rates of $E$. faecalis were $<60 \%$ for levofloxacin and $<25 \%$ for minocycline in Japan, whereas in Latin American regions, these were 6.1\% to $69.4 \%$ and $34.9 \%$ to $51.5 \%$, respectively. ${ }^{23}$ The susceptibility rates of $E$. faecium were $<30 \%$ for both levofloxacin and minocycline in Japan. Consequently, the 30-day mortality due to bacteremia was reportedly $21.4 \%$ and $34.6 \%$ in patients with $E$. faecalis and $E$. faecium, respectively. ${ }^{24}$ In contrast, previous studies showed that the mortality associated with non-faecium, non-faecalis enterococcal bloodstream infections was relatively low. ${ }^{25,26}$ Treatment failure for non-faecium/faecalis enterococcal bacteremia also occurred less frequently, suggesting their lower virulence relative to $E$. faecium and $E$. faecalis strains. ${ }^{27}$

We have identified EST as an independent risk factor for AC caused by E. faecalis and/or E. faecium. In this study, all patients who had undergone EST had a history of AC with antibiotic use (mostly cephalosporins). Consequently, E. faecalis and E. faecium, which have intrinsic resistance to cephalosporins, were likely to be detected as the pathogen for recurrent AC after EST.

Weber et al. ${ }^{16}$ reported that the isolation rates of Enterococcus spp. were significantly higher in cholangitis episodes with biliary endoprosthesis than in cholangitis 


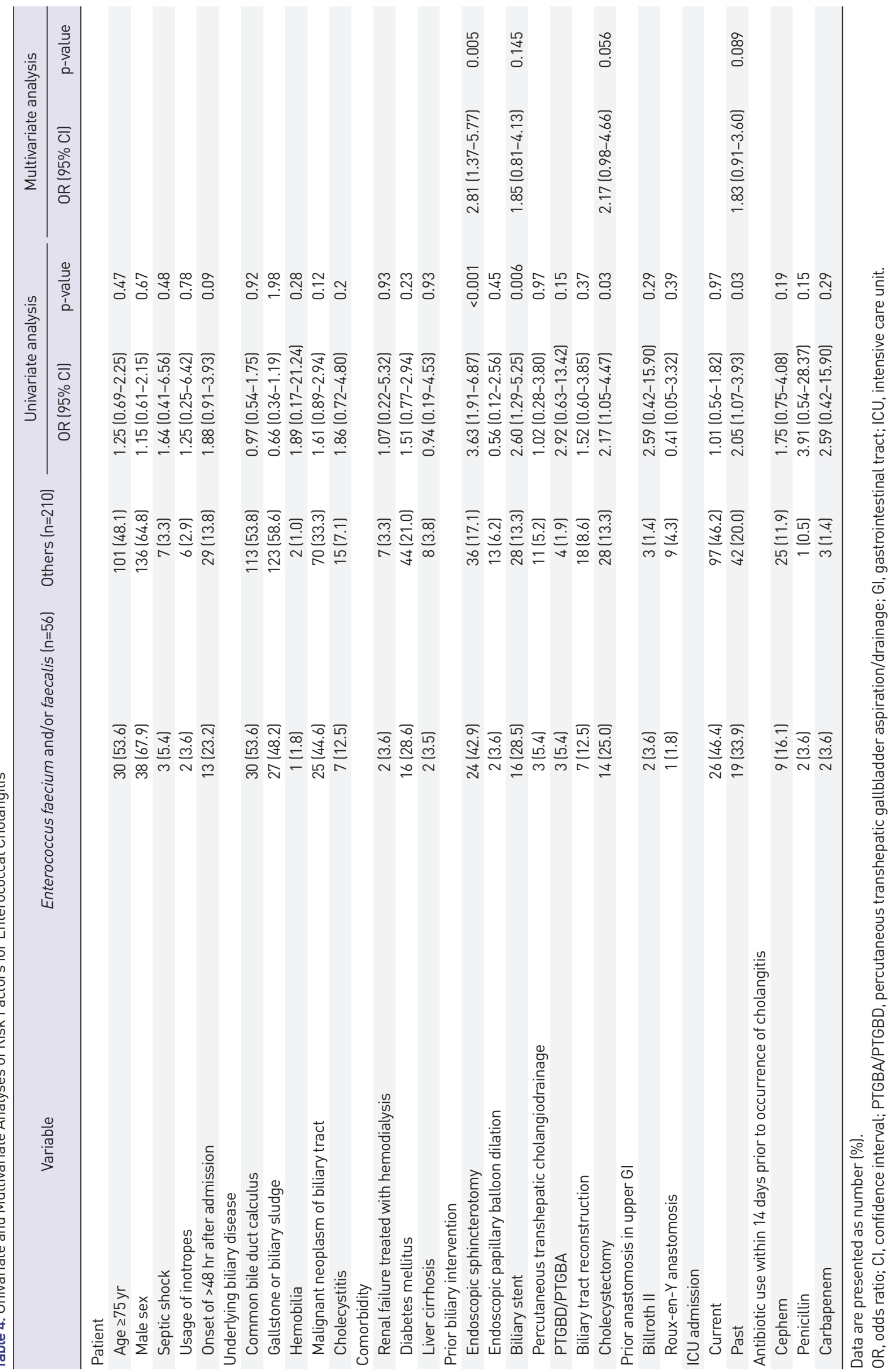


episodes without biliary endoprosthesis. They also reported that Pseudomonas aeruginosa and E. faecium were more frequently isolated from patients with a biliary endoprosthesis. In their study, most patients with biliary endoprosthesis may have been in a post-EST state, and our findings are comparable to their results. As EST and biliary stent are similar predictors, the multivariate analysis was recalculated excluding either variable. The odds ratio for EST was then superior to that of biliary stent (3.53 vs 3.01). In addition, most patients with biliary stent in our study had malignant neoplasm of the biliary tract. Therefore, they regularly consulted with physicians and were admitted to hospitals, and their AC was thus considered to be healthcare-associated. Overall, in our study, prior EST was considered as a common predictor. Moreover, E. faecalis is reportedly the second most predominant strain isolated from urinary catheter biofilms, following $P$. aeruginosa. ${ }^{28}$ Thus, these species are likely to colonize artificial objects and/or objects that developed after interventions took place, including micro-biliary sludge, or on the surface of the biliary tract.

In contrast to the post-EST state, no significant difference was observed between patients with and without EPBD. Both EST and EPBD are performed to dilate the duodenal papilla, but the function of the papilla after an intervention may be different because of the presence or absence of the cleavage of the sphincter muscle of the papilla. A previous study showed that the recurrence rate of common bile duct stones was higher in post-EST patients than in post-EPBD patients (26.3\% vs $6.3 \%)$, ${ }^{29}$ suggesting that $\mathrm{AC}$ is less likely to recur after EPBD than after EST. The dysfunction of Oddi may lead to the invasion of bacteria, including Enterococcus spp., into the bile duct.

Our study revealed that AC with prior EST was more likely to be caused by $E$. faecalis and/or $E$. faecium. Therefore, antibiotics with an activity against Enterococcus spp. have to be selected as an empirical therapy for AC patients after EST. Ampicillin is one of the candidates suitable for this purpose. Among cases caused by E. faecalis, susceptibility to ampicillin was $100 \%$ in Japan, ${ }^{30}$ the United States, and Europe, ${ }^{31}$ and $78 \%$ to $99 \%$ in Latin American regions. ${ }^{23}$ In contrast, among cases caused by $E$. faecium, susceptibility to ampicillin was lower, with $>60 \%$ in Japan, ${ }^{30} 0 \%$ to $7.9 \%$ in the United States and Europe, ${ }^{31}$ and $0.9 \%$ to $26 \%$ in Latin American regions. ${ }^{23}$ Therefore, we cannot treat patients with AC with EST or biliary endoprosthesis using third-generation cephalosporin alone, but should consider adding ampicillin treatment for patients with prior EST. In regions where ampicillin-resistant $E$. faecium is dominant, vancomycin, linezolid, or tigecycline should be considered for empirical antimicrobial treatment.
There are limitations to our current study. First, although we analyzed the first episodes of AC observed in our hospital in each patient, the episodes prior to their hospital admission could not be examined. Second, our research is a retrospective analysis of data obtained from a single center. Third, despite the fact that VRE is a serious problem worldwide, we were unable to investigate the risk factors of AC caused by VRE. Although our institution has access to equipment for isolating VRE, no such cases were encountered in our hospital owing to the rarity of VRE cases in Japan. Therefore, the generalizability of our results to regions with an increased prevalence of AC caused by VRE is limited. Future studies conducted in other regions where AC caused by VRE is dominant are warranted. Further, we detected causative pathogens in bile and/or blood cultures. Previously, patients with AC were significantly more likely to have positive bile cultures. ${ }^{32}$ Previous research has revealed that only $1.6 \%$ of blood cultures were impacted on management of patients in the emergency department. ${ }^{33}$ However, in the current study, for cases in which both bile and blood cultures were obtained, no significant difference in sensitivity between bile and blood cultures was observed.

In conclusion, the present study revealed that prior EST is an independent risk factor of AC caused by $E$. faecalis and/or $E$. faecium. To date, the therapeutic guidelines do not consider the existence of different bacterial spectra in patients with and without prior EST. Thus, clinicians should confirm the medical history of patients with AC for appropriate empirical therapy.

\section{CONFLICTS OF INTEREST}

No potential conflict of interest relevant to this article was reported.

\section{ACKNOWLEDGEMENTS}

We would like to thank Yuka Ono for accessing the biological database of Mitsui Memorial Hospital.

\section{AUTHOR CONTRIBUTIONS}

Study concept and design: Y.K., J.K., N.T. Data acquisition: Y.K., S.K., K.K., T.O., M.S., K.T. Data analysis and interpretation: Y.K., J.K., N.T. Drafting of the manuscript: Y.K. Critical revision of the manuscript for important intellectual content: J.K., N.T. Study supervision: J.K., N.T. 


\section{ORCID}

Yuki Karasawa

https://orcid.org/0000-0001-8278-7641

Jun Kato

https://orcid.org/0000-0002-1869-2135

Satoshi Kawamura

https://orcid.org/0000-0002-7031-5388

Kentaro Kojima https://orcid.org/0000-0002-2881-1679

Takamasa Ohki https://orcid.org/0000-0003-4275-468X

Michiharu Seki https://orcid.org/0000-0003-3131-0004

Kazumi Tagawa https://orcid.org/0000-0003-0148-8434

Nobuo Toda https://orcid.org/0000-0001-5426-1896

\section{REFERENCES}

1. Scott-Conner CE, Grogan JB. The pathophysiology of biliary obstruction and its effect on phagocytic and immune function. J Surg Res 1994;57:316-336.

2. Lipsett PA, Pitt HA. Acute cholangitis. Surg Clin North Am 1990;70:1297-1312.

3. Gigot JF, Leese T, Dereme T, Coutinho J, Castaing D, Bismuth $\mathrm{H}$. Acute cholangitis: multivariate analysis of risk factors. Ann Surg 1989;209:435-438.

4. Lee JG. Diagnosis and management of acute cholangitis. Nat Rev Gastroenterol Hepatol 2009;6:533-541.

5. Gomi H, Solomkin JS, Schlossberg D, et al. Tokyo Guidelines 2018: antimicrobial therapy for acute cholangitis and cholecystitis. J Hepatobiliary Pancreat Sci 2018;25:3-16.

6. Zimmer V, Lammert F. Acute bacterial cholangitis. Viszeralmedizin 2015;31:166-172.

7. Lee F, Ohanian E, Rheem J, Laine L, Che K, Kim JJ. Delayed endoscopic retrograde cholangiopancreatography is associated with persistent organ failure in hospitalised patients with acute cholangitis. Aliment Pharmacol Ther 2015;42:212-220.

8. Voigtländer T, Leuchs E, Vonberg RP, et al. Microbiological analysis of bile and its impact in critically ill patients with secondary sclerosing cholangitis. J Infect 2015;70:483-490.

9. Gargouri D, Ouakaa-Kchaou A, Kochlef A, et al. Microbiological study and antimicrobial susceptibility of bile in biliary therapeutic endoscopy. Tunis Med 2015;93:602-605.

10. Gomi H, Solomkin JS, Takada T, et al. TG13 antimicrobial therapy for acute cholangitis and cholecystitis. J Hepatobiliary Pancreat Sci 2013;20:60-70.

11. Wisplinghoff H, Bischoff T, Tallent SM, Seifert H, Wenzel RP, Edmond MB. Nosocomial bloodstream infections in US hospitals: analysis of 24,179 cases from a prospective nationwide surveillance study. Clin Infect Dis 2004;39:309-317.

12. Ramsey AM, Zilberberg MD. Secular trends of hospitalization with vancomycin-resistant enterococcus infection in the United States, 2000-2006. Infect Control Hosp Epidemiol 2009;30:184-186.
13. Watanabe S, Kobayashi N, Quiñones D, et al. Genetic diversity of the low-level vancomycin resistance gene vanC-2/vanC-3 and identification of a novel vanC subtype (vanC-4) in Enterococcus casseliflavus. Microb Drug Resist 2009;15:1-9.

14. Lodise TP, McKinnon PS, Tam VH, Rybak MJ. Clinical outcomes for patients with bacteremia caused by vancomycinresistant enterococcus in a level 1 trauma center. Clin Infect Dis 2002;34:922-929.

15. Reuken PA, Torres D, Baier M, et al. Risk factors for multidrug resistant pathogens and failure of empiric first-line therapy in acute cholangitis. PLoS One 2017;12:e0169900.

16. Weber A, Schneider J, Wagenpfeil S, et al. Spectrum of pathogens in acute cholangitis in patients with and without biliary endoprosthesis. J Infect 2013;67:111-121.

17. Buschelman BJ, Bale MJ, Jones RN. Species identification and determination of high-level aminoglycoside resistance among enterococci: comparison study of sterile body fluid isolates, 1985-1991. Diagn Microbiol Infect Dis 1993;16:119122.

18. Facklam RR, Collins MD. Identification of Enterococcus species isolated from human infections by a conventional test scheme. J Clin Microbiol 1989;27:731-734.

19. Gordon S, Swenson JM, Hill BC, et al. Antimicrobial susceptibility patterns of common and unusual species of enterococci causing infections in the United States. Enterococcal Study Group. J Clin Microbiol 1992;30:2373-2378.

20. Mondino SS, Castro AC, Mondino PJ, et al. Phenotypic and genotypic characterization of clinical and intestinal enterococci isolated from inpatients and outpatients in two Brazilian hospitals. Microb Drug Resist 2003;9:167-174.

21. Coombs GW, Pearson JC, Daly DA, et al. Australian Enterococcal Sepsis Outcome Programme annual report, 2013. Commun Dis Intell Q Rep 2014;38:E320-E326.

22. Hidron AI, Edwards JR, Patel J, et al. NHSN annual update: antimicrobial-resistant pathogens associated with healthcare-associated infections: annual summary of data reported to the National Healthcare Safety Network at the Centers for Disease Control and Prevention, 2006-2007. Infect Control Hosp Epidemiol 2008;29:996-1011.

23. Vega S, Dowzicky MJ. Antimicrobial susceptibility among Gram-positive and Gram-negative organisms collected from the Latin American region between 2004 and 2015 as part of the Tigecycline Evaluation and Surveillance Trial. Ann Clin Microbiol Antimicrob 2017;16:50.

24. Pinholt M, Ostergaard C, Arpi M, et al. Incidence, clinical characteristics and 30-day mortality of enterococcal bacteraemia in Denmark 2006-2009: a population-based cohort study. Clin Microbiol Infect 2014;20:145-151.

25. Pappas G, Liberopoulos E, Tsianos E, Elisaf M. Enterococcus casseliflavus bacteremia: case report and literature review. J Infect 2004;48:206-208. 
26. Tan CK, Lai CC, Wang JY, et al. Bacteremia caused by nonfaecalis and non-faecium Enterococcus species at a medical center in Taiwan, 2000 to 2008. J Infect 2010;61:34-43.

27. Choi SH, Lee SO, Kim TH, et al. Clinical features and outcomes of bacteremia caused by Enterococcus casseliflavus and Enterococcus gallinarum: analysis of 56 cases. Clin Infect Dis 2004;38:53-61.

28. Macleod SM, Stickler DJ. Species interactions in mixedcommunity crystalline biofilms on urinary catheters. J Med Microbiol 2007;56(Pt 11):1549-1557.

29. Chung JW, Chung JB. Endoscopic papillary balloon dilation for removal of choledocholithiasis: indications, advantages, complications, and long-term follow-up results. Gut Liver 2011;5:1-14.

30. Takesue Y, Kusachi S, Mikamo H, et al. Antimicrobial susceptibility of common pathogens isolated from postopera- tive intra-abdominal infections in Japan. J Infect Chemother 2018;24:330-340.

31. Mendes RE, Castanheira M, Farrell DJ, Flamm RK, Sader HS, Jones RN. Longitudinal (2001-14) analysis of enterococci and VRE causing invasive infections in European and US hospitals, including a contemporary (2010-13) analysis of oritavancin in vitro potency. J Antimicrob Chemother 2016;71:3453-3458.

32. Salvador VB, Lozada MC, Consunji RJ. Microbiology and antibiotic susceptibility of organisms in bile cultures from patients with and without cholangitis at an Asian academic medical center. Surg Infect (Larchmt) 2011;12:105-111.

33. Kelly AM. Clinical impact of blood cultures taken in the emergency department. J Accid Emerg Med 1998;15:254256. 